\title{
Reseña del libro El ABC y D de la formación docente. Desarrollo profesional docente desde la perspectiva de Vaillant y Marcelo
}

\author{
Review of the book The $A B C$ and $D$ of teacher training. Teacher professional \\ development from Vaillant and Marcelo's perspective
}

Bismar Galána ORCID: 0000-0001-9344-4425

Recibido: 08/04/2020 • Aprobado: 09/04/2020

Cómo citar: Galán, B. (2020). Reseńa del libro El ABC y D de la formación docente. Desarrollo profesional docente desde la perspectiva de Vaillant y Marcelo. Ciencia y Educación, 4(3), 169-171. Doi: https://doi.org/10.22206/cyed.2020.v4i3.pp169-171

La colección "Educación Hoy Estudios", de Narcea Ediciones, en la que se recogen obras fundamentales del pensamiento pedagógico y de la investigación educativa, publicó la obra $E l A B C$ y $D$ de la formación docente (2015), de los doctores Denise Vaillant y Carlos Marcelo. También, en el año 2009, en la referida colección, fue incluido el texto Desarrollo profesional docente. ¿Cómo se aprende a enseñar?, otro valioso trabajo de estos investigadores. Su más reciente obra conjunta, igualmente publicada por Narcea Ediciones, Hacia una formación disruptiva de docente (2018) demuestra el interés de los autores por el tema.

Denise Vaillant se ha destacado por sus reflexiones y análisis en el área de la formación docente. Sus aportaciones teóricas y áreas de estudio, desde su natal Uruguay, abarcan no solo el contexto latinoamericano. Además, la reforma e innovación educativas constituyen centro de múltiples artículos y libros suyos. Mientras Carlos Marcelo, catedrático de la Universidad de Sevilla, con permanente incursión en los espacios formativos de Latinoamérica, comparte línea de investigación y producción teórica con Vaillant, con importantes aportaciones al conocimiento científico en esta línea de trabajo, así como en las acciones de inducción de maestros nóveles y el aprendizaje en la sociedad del conocimiento, entre muchas otras aristas relacionadas con temas del área educativa. De manera que los autores realizan trabajos colaborativos de producción científica muy valiosos para estudios relacionados con la formación inicial y continua de los profesionales de la educación.

El libro que reseño en este texto está estructurado en cuatro partes, más la introducción y las conclusiones. El ABC y D lo justifica el hecho de que: la parte A se corresponde con Antecedentes, es decir, las etapas previas del oficio de enseñar; la B, con Base, que contempla la fase de formación inicial como punto

a Instituto de Formación Docente Salomé Ureña, República Dominicana

Correo-e: bismar.galan@isfodosu.edu.do 
de partida, los modelos más comunes, así como experiencias significativas; la parte $\mathrm{C}$, de Comienzo, habla de los primeros años de vida profesional y la etapa de inserción laboral. Mientras, la D, de Desarrollo, contempla lo referido a la formación continua del docente. De manera que, en los diez capítulos contenidos en estas partes, los autores van desde las peculiaridades de la enseñanza de adultos hasta el significado de las tecnologías y la conectividad en la formación del docente.

Todas las partes y capítulos de este libro merecen igual atención, dada la calidad de sus contenidos, coherencia y equilibrio. No obstante, destaco la cuarta parte (capítulos del 8 al 10), que recoge de manera específica las perspectivas de Vaillant y Marcelo respecto del "desarrollo profesional docente". Esta podría hacer las mayores aportaciones en las intenciones de ejecutar programas formativos que propicien las competencias que requieren los maestros para un eficiente desempeño de su labor.

En la introducción del capítulo 8, realizan un ponderado compendio del concepto de "desarrollo profesional docente" que asumen diferentes autores (Rudduck, 1991; Heideman, 1990; Fullan, 1990; Day, 1999; Bredeson, 2002 y Kelchtermans, 2004), lo que podría permitir la asunción de posiciones y la reconstrucción de nuevas conceptualizaciones alrededor del término. Además, suman su perspectiva de la interacción con los contextos espacial y temporal como determinante en el referido desarrollo.

En lo que han llamado "Una conceptualización inicial", dentro de este capítulo, destacan otros conceptos básicos relacionados con el tema: "profesionalización”, “autonomía profesional”, "práctica formativa”, entre otros, que llaman a establecer posiciones y relaciones de valor científico cuando se hace referencia a la preparación del maestro. Aquí critican el hecho de que los docentes no participen en el diseño de su formación, así como la falta de apropiación de los procesos y resultados de esa formación por parte de dichos actores de los sistemas educativos. Luego, presentan los principios que orientan el desarrollo profesional desde la perspectiva de Hawly y Valli (1998).

Considero que una de las aportaciones más importantes en este capítulo 8 del libro es lo relativo a los elementos básicos para explicar la calidad del desarrollo profesional docente (DPD): contenido, proceso y contexto, y que sintetizan en la "Figura 8.1. El DPD y los aprendizajes de los estudiantes".

Lo concerniente a la evaluación del desarrollo profesional es el último tema de este capítulo 8 , donde Vaillant y Marcelo comparten los cinco momentos que aporta el modelo de evaluación de Guskey (2000). Asimismo, apuntalan la idea de asumir el desarrollo profesional centrado en los trabajos de los estudiantes dada la certeza de que de esa manera se logra el desarrollo de sus competencias.

El resto de los capítulos de la cuarta parte patentizan el valor que los investigadores otorgan a la ejecución de la formación. El capítulo 9 hace referencia a la innovación en esta área, con ejemplos del hecho en diferentes regiones del mundo: sudeste asiático, Estados Unidos, España y Latinoamérica. Es plausible ver en el capítulo 10 la inclusión del uso de las tecnologías en los procesos formativos y su incidencia en las prácticas docentes y, por consiguiente, los cambios e innovaciones que genera en el área educativa.

De manera que, las aportaciones contenidas en este texto y de modo específico en su cuarta parte, podrían ser aprovechadas en nuevas indagaciones sobre el tema y como valiosas herramientas conceptuales para llevar a la práctica acciones concretas relacionadas y/o dirigidas a la formación docente. El lenguaje claro, preciso y coherente con que se ha presentado cada idea constituye un distintivo más de esta obra que seguramente resultará de interés para los investigadores del tema, para los docentes y para los tomadores de decisiones respecto de cómo lograr un desarrollo profesional que se traduzca en transferencia de saberes a los estudiantes. La amplia bibliografía incluida en el libro permitirá profundizar en temas específicos que resulten de interés al lector. 


\section{Referencias}

Bredeson, P. (2002). The architecture of professional development: materials, messages and meaning. International Journal of Educational Research, 37(8), 661-675.

Day, C. (1999). Developing Teachers. The Challenges of Lifelong Learning. London: Falmer Press.

Fullan, M. (1990). Staff Development Innovation and Institutional Development. En B. Joyce (ed.). School Culture Through Staff Development (pp. 3-25). Virginia: ASCD.

Guskey, T. (2000). The characteristics of effective professional development: A synthesis of lists: ERIC ED, 478380.

Hawly, W. y Valli, L. (1998). The Essentials of Effective Professional Development. A New Consensus. En L. Darling-Hammond y G. Sykes (eds.). Teaching as the learning profession. Handbook of Policy and Practice (pp. 127-149). San Francisco: Jossey-Bass.
Heideman, C. (1990). Introduction to staff development. En P. Burke (Ed.). Programming for staff development (pp. 3-9). London: Falmer Press.

Kelchtermans, G. (2004). CPD for professional renewal: moving beyond knowledge for practice. En C. Day y J. Sachs (Ed.). International Handbook on the Continuing Professional Development of Teachers. Berkshire: McGraw-Hill Education.

Rudduck, J. (1991). Innovation and Change. Milton Keynes: Open University.

Vaillant, D. y Marcelo, C. (2015). El ABC y D de la formación docente. Madrid: Narcea. 